\title{
RENDIMENTO DOS PRODUTOS DA PIRÓLISE DE LODO DE ESGOTO ANAERÓBIO EM REATOR DE LEITO FIXO EM DIFERENTES TEMPERATURAS
}

\author{
L. F. TEIXEIRA ${ }^{1}$, G. E. G. VIEIRA ${ }^{1}$, L. A. R. CORREIA ${ }^{2}$, A. G. N. $\operatorname{COLEN}^{1}$, G. L. de \\ AGUIAR Jr. ${ }^{1}$ \\ ${ }^{1}$ Universidade Federal do Tocantins (UFT), Laboratório de Ensaio e Desenvolvimento em \\ Biomassa e Biocombustíveis (LEDBIO) \\ ${ }^{2}$ Instituto Federal de Educação, Ciência e Tecnologia do Tocantins (IFTO) \\ E-mail para contato: glauciaeliza@uft.edu.br
}

\begin{abstract}
RESUMO - O aumento populacional aliado à melhoria da eficiência do tratamento de esgoto implica diretamente o aumento da produção do lodo de esgoto, que é o principal resíduo gerado nas estações de tratamento de esgotos. A pirólise é uma tecnologia promissora que possibilita a produção de quatro frações quando aplicada ao lodo residual: bio-óleo, fração aquosa, fração sólida e gasosa, com potencial combustível e utilização na indústria química. Este artigo tem como objetivo avaliar o processo de pirólise aplicado ao lodo de esgoto anaeróbio em diferentes temperaturas, favorecendo a otimização das condições do processo para maximizar o rendimento do produto desejado. Foi realizada a caracterização físicoquímica do lodo (umidade, voláteis, cinzas e carbono fixo) e aplicação da pirólise em reator de leito fixo em escala laboratorial nas temperaturas de $450^{\circ} \mathrm{C}, 500^{\circ} \mathrm{C}$ e $550^{\circ} \mathrm{C}$. O lodo de esgoto apresentou teor de umidade de $6,85 \%$, voláteis $54,99 \%$, cinzas $33,33 \%$ e carbono fixo $4,83 \%$. O maior rendimento de bio-óleo de $15,6 \%$ foi obtido à $550^{\circ} \mathrm{C}$, seguido pelo rendimento de $15,03 \%$ e $13,83 \%$, respectivamente nas temperaturas $500^{\circ} \mathrm{C}$ e $450^{\circ} \mathrm{C}$, já os valores de rendimento de fração sólida foram $56,52 \%, 59,73 \%$ e $60,97 \%$, respectivamente a $550^{\circ} \mathrm{C}, 500^{\circ} \mathrm{C}$ e $450^{\circ} \mathrm{C}$.
\end{abstract}

\section{INTRODUÇÃO}

O lodo de esgoto é o principal resíduo gerado pelas estações de tratamento de esgotos domésticos (Fonts et al., 2012). Sua disposição enfrenta uma série de problemas ambientais relacionados às emissões atmosféricas, risco a saúde pública e contaminação do solo e dos recursos hídricos (Aggelakis et al., 2005). Atualmente as principais formas de destinação ao lodo de esgoto são o uso agrícola, a incineração e a disposição em aterros (Fonts et al., 2012).

Em um contexto global, acredita-se que a produção de lodo de esgoto nas próximas décadas continuará aumentando gradualmente, considerando o desenvolvimento da população, urbanização e industrialização (Cao; Pawlowski, 2012). Essa perspectiva vem incentivando nos últimos anos, a busca e aplicação de tecnologias que visem seu melhor aproveitamento (Fonts et al., 2009).

O lodo de esgoto é abundante em matéria volátil e tem sido considerado um potencial recurso como matéria-prima, despertando interesse em sua utilização através de processos de 
conversão em uma diversidade de produtos tais como, produtos químicos e combustíveis através de processos termoquímicos (Hu et al., 2014).

Diversas tecnologias, como a pirólise e a gaseificação, têm sido desenvolvidas e representam alternativas viáveis para o uso de lodo residual (Pedroza et al., 2014). A pirólise tem recebido maior atenção nos últimos anos, como uma rota aceitável e sendo adotada como uma forma vantajosa para eliminação de resíduos orgânicos industriais e urbanos, considerando seu crescente e positivo desenvolvimento para produção de combustíveis (Bridgwater, 2012).

A pirólise pode ser definida como um processo de degradação térmica de qualquer material orgânico, na ausência de um agente oxidante (Vieira, 2004). Durante a pirólise são gerados produtos líquidos (bio-óleo e fração aquosa), gasosos e sólidos, que são produzidos em diferentes proporções a partir do rompimento das ligações, levando à obtenção de produtos com capacidade energética e propriedades melhores do que aquelas da biomassa inicial (Vieira, 2004). Essa possibilidade de obter produtos de maior valor agregado torna esse processo uma opção muito atraente diante das iniciativas atuais de busca de novas fontes renováveis para a produção de energia mais limpa (Gómez, 2002).

A temperatura, a taxa de aquecimento e o tempo de residência da biomassa são os principais parâmetros que influenciam significativamente a composição e o rendimento dos produtos obtidos (Bridgwater, 2003; Bridgwater, 2012; Fonts et al., 2012; Vieira et al., 2009).

Este trabalho tem como objetivo analisar o processo de pirólise aplicado ao lodo de esgoto anaeróbio com adição de $5 \%$ de $\mathrm{Fe}_{2} \mathrm{O}_{3}(\mathrm{~m} / \mathrm{m})$ em diferentes parâmetros de temperatura $\left(450^{\circ} \mathrm{C}, 500^{\circ} \mathrm{C}\right.$ e $\left.550^{\circ} \mathrm{C}\right)$ favorecendo a otimização das condições do processo para maximizar o rendimento do produto de interesse.

\section{MATÉRIAIS E MÉTODOS}

\subsection{Coleta do lodo de esgoto}

As amostras de lodo de esgoto foram obtidas na Estação de Tratamento de Esgotos Norte (ETE Norte), na cidade de Palmas-TO. Foram coletadas na válvula de descarga de um reator UASB (Upflow Anaerobic Sludge Blanket) de acordo com as normas técnicas NBR 10.007 e NBR 10.004.

\subsection{Preparo do lodo de esgoto}

A secagem das amostras de lodo de esgoto foi realizada em forno solar artesanal até peso constante. Em seguida foram trituradas em moinho, homogeneizadas em peneira com abertura de $0,355 \mathrm{~mm}$ e adicionado catalisador $\mathrm{Fe}_{2} \mathrm{O}_{3}$, da marca cromoline, na proporção $5 \%$ $(\mathrm{m} / \mathrm{m})$. 


\subsection{Caracterização físico-química do lodo de esgoto}

O lodo de esgoto foi caracterizado através das analises de teor de umidade, sólidos voláteis, cinzas e carbono fixo de acordo com os respectivos métodos descritos na Tabela 1.

Tabela 1 - Métodos e fórmulas utilizadas na caracterização físico-química do lodo de esgoto

\begin{tabular}{|l|c|}
\hline \multicolumn{1}{|c|}{ Análise } & Método \\
\hline Teor de umidade & ASTM D3173-85 \\
\hline Teor de sólidos voláteis & ASTM D2415-66 \\
\hline Teor de cinzas & ASTM D2415-66 \\
\hline Teor de carbono fixo & Por diferença \\
\hline
\end{tabular}

\subsection{Processo de pirólise}

O processo em batelada foi realizada em reator de leito fixo da marca EDG modelo FTHI bipartido, o sistema que compõe a unidade de pirólise em escala laboratorial do LEDBIO (Laboratório de ensaio e desenvolvimento em biomassas e biocombustíveis), da Universidade Federal do Tocantins, é representado pelo esquema na Figura 1.

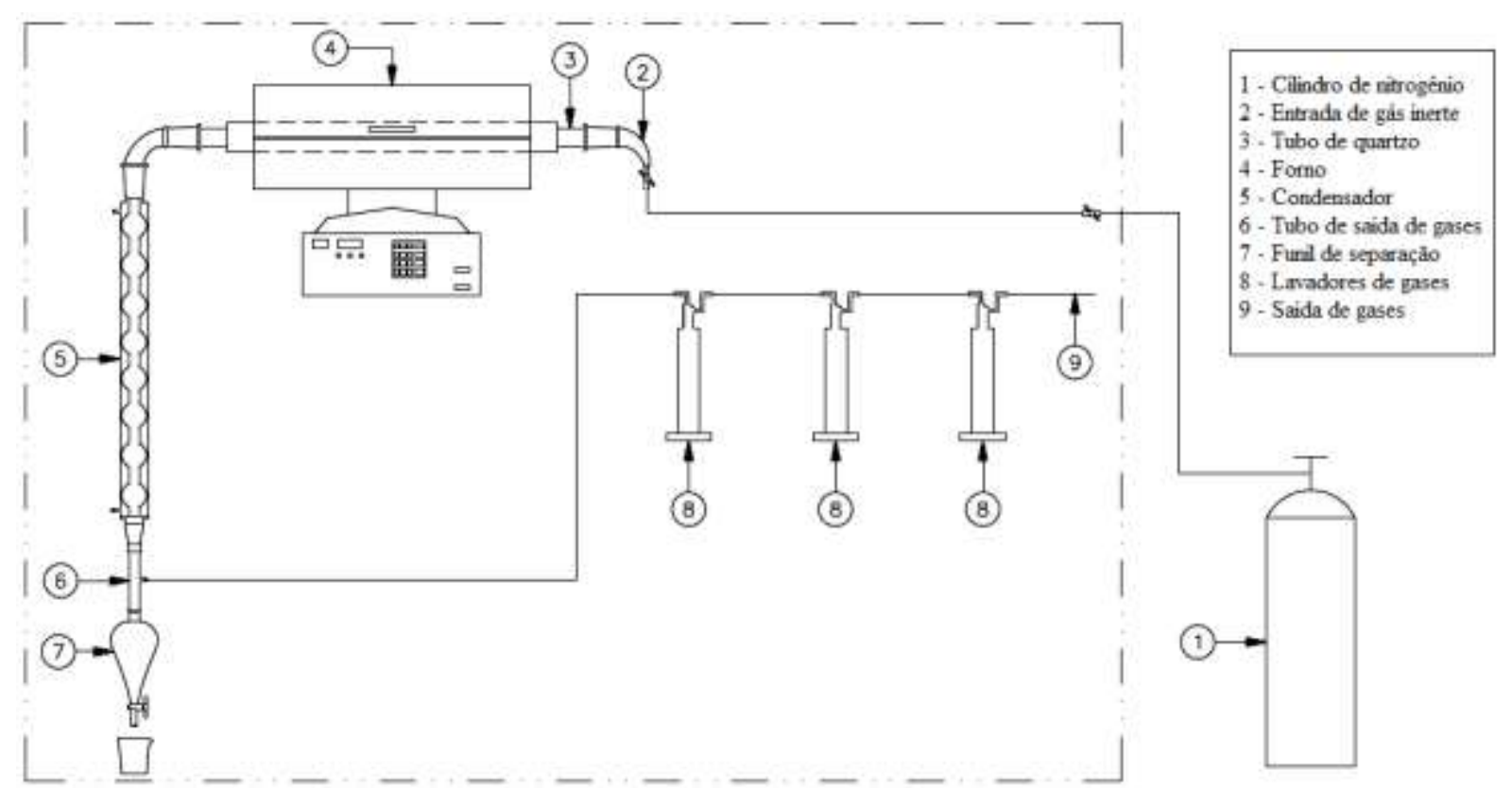

Figura 1 - Desenho esquemático da unidade de pirólise em escala laboratorial Adaptado de CORREIA (2013)

Foi utilizada uma alíquota de 40 gramas da amostra no processo de pirólise, que foi realizado com uma taxa de aquecimento de $10^{\circ} \mathrm{C} \cdot \mathrm{min}^{-1}$, tempo de residência de 180 minutos e utilizando três temperaturas: $450^{\circ} \mathrm{C}, 550^{\circ} \mathrm{C}$ e $550^{\circ} \mathrm{C}$. 


\section{RESULTADOS E DISCUSSÕES}

A caracterização físico-química do lodo é fundamental para aplicação desse material em processos termoquímicos visando produtos com potencial energético, segue na Tabela 2 seus resultados.

Tabela 2 - Caracterização físico-química do lodo de esgoto

\begin{tabular}{|c|c|c|c|c|}
\hline $\begin{array}{c}\text { Umidade } \\
(\%)\end{array}$ & $\begin{array}{c}\text { Cinzas } \\
(\%)\end{array}$ & $\begin{array}{c}\text { Sólidos voláteis } \\
(\%)\end{array}$ & $\begin{array}{c}\text { Carbono fixo } \\
(\%)\end{array}$ & Literatura \\
\hline 6,85 & 34,7 & 58,8 & 4,83 & Presente trabalho \\
\hline 6,8 & 32,4 & 59,2 & 8,4 & Sanchez et al. $(2009)$ \\
\hline 6,0 & 38,1 & 53,9 & 2,0 & Pedroza et al. $(2014)$ \\
\hline $2,8-6,3$ & $30,3-39,7$ & $53,3-58,1$ & $2,1-6,7$ & Silva $(2012)$ \\
\hline $1,5-7,1$ & $22,6-52,0$ & $38,3-66,8$ & $0,8-19,7$ & Fonts et al. $(2012)$ \\
\hline $11,5-24,2$ & $45,0-46,2$ & $25,7-40,5$ & $1,94-5,25$ & Leal $(2010)$ \\
\hline 5,30 & 47,5 & 53 & - & Pokorna et al. $(2009)$ \\
\hline
\end{tabular}

Fatores como condições climáticas, sazonalidade, forma de tratamento e coleta interferem diretamente no teor de umidade (Correia et al., 2012). A determinação do teor de umidade da amostra desempenha um relevante papel em processos de pirólise, devido à influência que a umidade exerce durante o processo térmico (Borges et al,. 2008). Pois, quanto maior o teor de umidade presente na biomassa, maior será o gasto energético, implicando em um aumento no custo total de processamento, conduzindo, assim, à necessidade de secagem prévia da amostra (Colen, 2011).

O conhecimento do teor de sólidos voláteis é de grande importância no processo de pirólise, os mesmos são constituídos por matéria orgânica, que sofre desorção na primeira etapa da pirólise, e posteriormente passam pelo craqueamento e rearranjo químico, estágio de reações radicalares, de adição, hidrogenação e moleculares, formando novos compostos químicos que constituirão o bio-óleo ao serem condensados (Vieira, 2004). O alto valor de sólidos voláteis indica o alto percentual de substâncias orgânicas agregadas que volatilizam até $550^{\circ} \mathrm{C}$.

O teor de cinzas refere-se à presença de substâncias inorgânicas no lodo de esgoto e apresenta uma enorme influência sobre a pirólise do lodo de esgoto, em que a maior quantidade do teor de cinzas favorece um aumento do rendimento da fração sólida e gasosa e a diminuição da fração líquida, em comparação a um lodo com teor de cinzas baixo (Alexandre, 2013; Fonts et al., 2009). Pode ser atribuído ao teor de cinzas as reações de desidrogenação, favorecidas pelo seu efeito catalítico na pirólise (Silva, 2012). As características dos lodos de esgotos são bastante variáveis decorrendo de hábitos da população ou a possibilidade de mistura de efluentes domésticos e industriais. 
Os rendimentos dos produtos obtidos a partir do processo de pirólise de lodo de esgoto são apresentados na Tabela 3.

Tabela 3 - Rendimento médio das frações obtidos na pirólise de lodo de esgoto

\begin{tabular}{|c|c|c|c|c|}
\hline \multirow{2}{*}{ Temperatura $\left({ }^{\circ} \mathrm{C}\right)$} & \multicolumn{4}{|c|}{ Frações } \\
\cline { 2 - 5 } & Bio-óleo & Aquosa & Sólida & Gasosa \\
\hline 450 & 13,83 & 12,06 & 60,97 & 13,14 \\
\hline 500 & 15,03 & 12,90 & 59,73 & 12,34 \\
\hline 550 & 15,60 & 13,28 & 56,52 & 14,60 \\
\hline
\end{tabular}

* Valores da fração gasosa obtida por diferença

Tabela 4 - ANOVA para produção de bio-óleo a partir da pirólise de lodo de esgoto

\begin{tabular}{|c|c|c|c|c|c|c|}
\hline Fonte da variação & S.Q. & G. L. & Q. M. & F & valor-P & F crítico \\
\hline Entre grupos & 4,882222 & 2 & 2,441111 & 7,0313 & 0,026748 & 5,14325285 \\
\hline Dentro dos grupos & 2,083067 & 6 & 0,347178 & & & \\
\hline Total & 6,965289 & 8 & & & & \\
\hline
\end{tabular}

S.Q.: Soma de quadrados; G.L.: Grau de liberdade; Q.M.: Quadrado médio

Tabela 5 - ANOVA para produção de fração aquosa a partir da pirólise de lodo de esgoto

\begin{tabular}{|l|r|r|r|r|r|r|}
\hline \multicolumn{1}{|c|}{ Fonte da variação } & \multicolumn{1}{|c|}{ S.Q. } & G. L. & Q. M. & F & valor-P & F crítico \\
\hline Entre grupos & 2,3384 & 2 & 1,1692 & 20,21671 & 0,002158 & 5,143253 \\
\hline Dentro dos grupos & 0,347 & 6 & 0,057833 & & & \\
\hline Total & 2,6854 & 8 & & & & \\
\hline
\end{tabular}

S.Q.: Soma de quadrados; G.L.: Grau de liberdade; Q.M.: Quadrado médio

Tabela 6 - ANOVA para produção de fração sólida a partir da pirólise de lodo de esgoto

\begin{tabular}{|l|c|r|c|c|c|c|}
\hline Fonte da variação & S.Q. & G. L. & Q. M. & F & valor-P & F crítico \\
\hline Entre grupos & 31,59316 & 2 & 15,79658 & 38,63293 & 0,000374 & 5,143253 \\
\hline Dentro dos grupos & 2,453333 & 6 & 0,408889 & & & \\
\hline Total & 34,04649 & 8 & & & & \\
\hline
\end{tabular}

S.Q.: Soma de quadrados; G.L.: Grau de liberdade; Q.M.: Quadrado médio

Tabela 7 - ANOVA para produção de fração gasosa a partir da pirólise de lodo de esgoto

\begin{tabular}{|c|c|c|c|c|c|c|}
\hline Fonte da variação & S.Q. & G. L. & Q. M. & $\mathrm{F}$ & valor-P & F crítico \\
\hline Entre grupos & 7,829689 & 2 & 3,914844 & 2,340201 & 0,177293 & 5,143253 \\
\hline Dentro dos grupos & 10,0372 & 6 & 1,672867 & & & \\
\hline Total & 17,86689 & 8 & & & & \\
\hline
\end{tabular}

A análise estatística, apresentada pelas tabelas 4, 5, 6 e 7, mostram que houve diferença significativa entre médias na produção de bio-óleo, fração aquosa e sólida, a um nível de 5\% de significância. Os dados mostram que, o aumento da temperatura de $450^{\circ} \mathrm{C}$ a $500^{\circ} \mathrm{C}$ favoreceu o aumento das frações bio-óleo e aquosa. Esse aumento pode ser atribuído à devolatização da matéria orgânica e gaseificação parcial de resíduos carbonosos no carvão em temperaturas elevadas, o comportamento dos compostos da biomassa, durante a devolatilização, é resultado da soma dos componentes individuais do lodo, como celulose, hemicelulose, lignina, ácidos húmicos e hidrocarbonetos sólidos (Vieira 2004; Bridgwater, 
2007). A presença de componentes lignocelulósicos no lodo está relacionada com as características da fonte de esgoto. Níveis mais elevados de celulose e lignina são observados em lodo de esgoto de uma população cuja dieta é rica em fibras (Pedroza, 2014).

Inguanzo et al. (2002) observou que o rendimento da fração bio-óleo e aquosa foi maior quando a temperatura foi acrescida de $450^{\circ} \mathrm{C}$ para $650^{\circ} \mathrm{C}$, acima desta temperatura não foi observado aumento das frações. Silva (2012) realizou pirólise de lodo de esgoto sem a adição de catalisador, em condições semelhantes, e obteve os rendimentos de bio-óleo de 11,6 e $11,4 \%$, respectivamente nas temperaturas de processo de $450^{\circ} \mathrm{C}$ e $550^{\circ} \mathrm{C}$.

Parte da umidade presente no lodo residual pirolisado pode ter sofrido condensação para a fração líquida, formando a fração aquosa. De acordo com Sánchez et al. (2009) a formação da fração aquosa durante a pirólise ocorre devido à quebra dos grupos funcionais dos compostos voláteis durante as reações secundárias. O conteúdo de $\mathrm{OH}$ na amostra de lodo pode levar a uma maior ou menor formação da fração aquosa. Quando a quantidade desses grupos na amostra de lodo é alta, pode haver uma maior formação de água na fração líquida gerada no processo de degradação térmica.

O decréscimo na fração sólida na faixa de $450^{\circ} \mathrm{C}$ a $550^{\circ} \mathrm{C}$ pode ser atribuído ainda, a um aumento da volatilização dos hidrocarbonetos sólidos no carvão, assim como, pode ser atribuído à possível gaseificação parcial do resíduo de carbono (Fonts et al., 2009; Sanchez et $a l .$, 2009). Pattiya (2011) relatou que a redução do rendimento de fração sólida com o aumento da temperatura pode ser devido ao maior decomposição primária da biomassa em temperatura mais alta e/ou decomposição térmica secundária do carvão formado, antes de ser arrastada para fora da zona de reação. A fração gasosa não apresentou diferença significativa entre as médias pela analise de variância. $\mathrm{O}$ aumento do rendimento de gás com o aumento temperatura é possivelmente devido a uma combinação de reações secundárias, como o craqueamento (Pedroza, 2011).

\section{CONCLUSÃO}

A caracterização das amostras de no lodo de esgoto, obtidas em uma estação de tratamento de esgotos em Palmas - TO, apresentaram alto de teor de sólidos voláteis $(58,8 \%)$ e cinzas $(34,7 \%)$, e baixo teor de umidade $(6,8 \%)$ e carbono fixo $(4,83 \%)$.

Na pirólise do lodo de esgoto pode-se observar que o ensaio realizado com temperatura de processo de $550^{\circ} \mathrm{C}$ foi possível obter um maior rendimento de bio-óleo, fração aquosa e gasosa, ao passo que, diminui o rendimento de fração sólida.

Para maior rendimento de fração sólida foi observado melhor rendimento utilizando menores temperaturas, sendo na temperatura de $450^{\circ} \mathrm{C}$ o rendimento mais alto da fração sólida, que pode ser utilizado para produção de carvão ativado. 


\section{REFERÊNCIAS BIBLIOGRÁFICAS}

AGGELAKIS, A.; VOURVAXI, K.; DIAVATIS, I.; EFMORFOPOULOU, A.; KARTSONAS, N.; MAMAIS, D.; BOSDOGIANNI, A.; STAMOU, A. Alternative Management Methods of Wastewater Treatment Plants Byproducts. Technical Chamber of Greece, Athens 2005.

ALEXANDRE, G. P. Avaliação da produção de bio-óleo por termocatálise com óxido de cálcio de lodo de esgoto doméstico. 2013. 121 f. Dissertação (Mestrado) - Curso de Agroenergia. Universidade Federal do Tocantins. Palmas, 2013.

BORGES, F.; SELLIN, N.; MEDEIROS, S. H. W. Caracterização e avaliação de lodos de efluentes sanitário e industrial como biomassa na geração de energia. Ciência e Engenharia. v. 17 , p. $27-32.2008$.

BRIDGWATER, A. V.. Renewable fuels and chemicals by thermal processing of biomass. Chem. Eng. J., v. 91, p.87-102, 2003.

BRIDGWATER. A. V. Biomass pyrolysis. Biomass and Bioenergy, v. 31. p. 7 - 18, 2007.

BRIDGWATER, A. V. Review of fast pyrolysis of biomass and product upgrading. Biomass and Bioenergy. v. 38, p. 68 - 94, 2012.

CAO, Y.; PAWLOWSKI, A. Sewage sludge-to-energy approaches based on anaerobic digestion and pyrolysis: Brief overview and energy efficiency assessment. Ren. and Sust. En. Reviews., v. 16, p. 1657-1665, 2012.

COLEN, A. G. N. Caracterização físico-química e química do lodo de esgoto para aplicação como fonte de energia. 2011. 159 f. Dissertação (Mestrado) - Curso de Agroenergia, Universidade Federal do Tocantins, Palmas, 2011.

CORREIA, L. A. R. Estudo do processo de pirólise para o aproveitamento sustentável de lodo digerido doméstico. 2013. 134 f. Mestrado (Dissertação). Agroenergia. Universidade Federal do Tocantins. Palmas, 2013.

FONTS, I.; KUOPPALA, E.; OASMAA, A. Physicochemical properties of product liquid from pyrolysis of sewage sludge. Energy \& Fuels, v. 23, p. 4121-4128, 2009.

FONTS, I.; GEA, G.; AZUARA, M.; ÁBREGO, J.; ARAUZO, J. Sewage sludge pyrolysis for liquid production: A review. Ren. and Sust. En. Reviews, v. 16, p. 2781 -2805, 2012.

GÓMEZ, E. O. Estudo da pirólise rápida de capim elefante em leito fluidizado borbulhante mediante caracterização dos finos de carvão. 2002. 412 f. Tese (doutorado). Curso de Engenharia Agrícola. Faculdade de Engenharia Agrícola, Universidade Estadual de Campinas. Campinas, SP. 2002.

INGUANZO, M.; DOMÍNGUEZ, A.; MENÉNDEZ, J. A.; BLANCO, C. G.; PIS, J. J. On the pyrolysis of sewage sludge: the influence of pyrolysis conditions on solid, liquid and gas fractions. Journal of Analytical and Applied Pyrolysis, v. 63, n.1, p. 209-222, 2002. 
HU, Y.; LI, G.; YAN, M.; PING, C.; REN, J.; Investigation into the distribution of polycyclic aromatic hydrocarbons (PAHs) in wastewater sewage sludge and its resulting pyrolysis biooils. Science of the Total Environment, p. 459-464, 2014.

LEAL, E. R. M. Aplicação do processo de pirólise lenta ao lodo de esgoto adicionado de óxido de cálcio e ferro para obtenção de bio-óleo combustível. 2010. 257 f. Dissertação (Mestrado). Agroenergia. Universidade Federal do Tocantins, Palmas, 2010.

PATTIYA, A. Bio-oil production via fast pyrolysis of biomass residues from cassava plants in a fluidised-bed reactor. Bioresource Technology, v. 102, p. 1959-1967, 2011.

PEDROZA, M. M. Bio-óleo e bio-gás da degradação termoquímica de lodo de esgoto doméstico em cilindro rotativo. 2011. 237 f. Tese (Doutorado). Engenharia Química. Universidade Federal do Rio Grande do Norte. Natal, 2011.

PEDROZA, M. M.; SOUSA, J. F.; VIEIRA, G. E. G.; BEZERRA, M. B. D. Characterization of the products from the pyrolysis of sewage sludge in $1 \mathrm{~kg} / \mathrm{h}$ rotating cylinder reactor. J.of Analyt. and Ap. Py., v. 105, p. 108-115 2014.

POKORNA, E.; POSTELMANS, N.; JENICEK, P.; SCHREURS, S.; CARLEER, R.; YPERMAN, J. Study of bio-oils and solids from flash pyrolysis of sewage sludges. Fuel. v. 88. p. 1344-1350, 2009.

SANCHEZ, M. E.; MENÉDEZ, J. A.; DOMÍNGUEZ, A.; PIS, J. J.; MARTÍNEZ, O.; CALVO, L. F,; BERNAD, P. L. Effect of pyrolysis temperature on the composition of the oils obtained from sewage sludge. Biomassa and Bioenergy, v. 33, p. 933-940, 2009.

SILVA, L. C. A. Estudo do processo de pirólise de lodo de esgoto em reator de leito fixo em escala laboratorial. 2012. 67 f. Dissertação (Mestrado em Agroenergia). Universidade Federal do Tocantins. Palmas, 2012.

VIEIRA, G. E. G. Fontes alternativas de energia - Processo aperfeiçoado de conversão térmica. Tese (Doutorado). 2004. 181 f. Universidade Federal Fluminense. Rio de Janeiro, 2004.

VIEIRA, G. E. G., ROMEIRO, G. A., SELLA, S. M., DAMASCENO, R. N., PEREIRA, R. G. Low temperature conversion (LTC) - An alternative method to treat sludge generated in an industrial wastewater treatment station - Batch and continuous process comparison.

Bioresource Technology. v. 100, p.1544-1547, 2009. 